REVISTA MATEMÁTICA de la

Universidad Complutense de Madrid

Volumen 9, número 2: 1996

http://dx.doi.org/10.5209/rev_REMA.1996.v9.n2.17585

\title{
Unknotting number and knot diagram
}

\author{
Yasutaka NAKANISHI
}

\begin{abstract}
This note is a continuation of [N1], where we have discussed the unknotting number of knots with respect to knot diagrams. We will show that for every minimum-crossing knot-diagram among all unknotting-number-one two-bridge knot there exist crossings whose exchange yields the trivial knot, if the third Tait conjecture is true.
\end{abstract}

\section{Introduction}

The unknotting number $u(k)$ is defined to be the minimum number of exchanges of over- and under-crossing required to convert $k$ into the trivial knot over all knot diagrams representing $k$. The unknotting number is intuitive and attractive for knot theorists and there is no algorithm to compute it for a given knot until now. Our motivation is to find an approach. For a given knot, there are finite number of minimum crossing knot diagrams. If the condition "all knot diagrams" is restricted to "all minimum crossing knot diagrams", then we cannot obtain the unknotting numbers as in [N1] and [B]: The minimum crossing diagram of the knot $10_{8}$ as in Fig. 1 (a) (or 514 in the Conway notation [C]) is unique up to homeomorphism of projected 2-sphere, where exchanges of two crossings never yield the trivial knot, however the unknotting number of $10_{8}$ is two. (There is another diagram of the knot $10_{8}$ where exchanges

1991 Mathematics Subject Classification. 57M25.

Servicio Publicaciones Univ. Complutense. Madrid, 1996. 
of two erossings yield the trivial knot). The author is afraid that this situation is not exceptional. He has raised a question: Let $K$ be a minimum crossing knot diagram of a knot $k$. Can we convert $K$ into $K^{\prime}$ with $u\left(k^{\prime}\right)<u(k)$ by exchange of a croising? If the answer is affirmative for all knots, we can give an algorithm. For the diagram as in Fig. 1 (a), we can convert the knot $10_{8}$ into $6_{2}$ with $u\left(6_{2}\right)=1$ by exchange of the croissing marked by the asterisk.

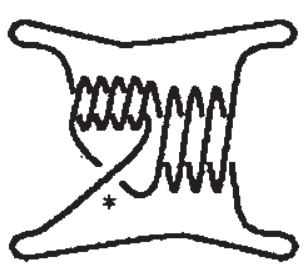

(a)

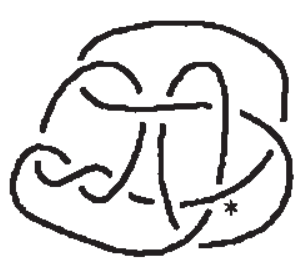

(b)

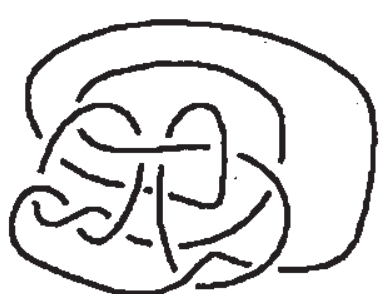

(c)

Fig. 1

In the above, it is necessary that $K$ is a minimum crossing diagram: Fig. 1 (b) and (c) illustrate two diagram of the famous Conway 11 crossing knot. The central diagram (b) has the minimum number of crossings, and exchange of the crossing marked by the asterisk yields the trivial knot. The right one (c) is not a minimum crossing diagram, and there is no crossing whose exchange yields the trivial knot. In fact, it is seen in [N2] that, for any unknotting-number-one knot, there exist a diagram where is no crossing whose exchange yields the trivial knot.

In this note, we give an affirmative answer for the class of unknottingnumber-one two-bridge knots. Kanenobu and Murakami [KM] characterized the unknotting-number-one two-bridge knots as knots represented in the Conway notation by

$$
C\left(a, a_{1} . a_{2}, \cdots, a_{n}, \pm 2,-a_{n} \cdots,-a_{2},-a_{1}\right) \text {. }
$$


In section 2 , we deform the diagram above into the alternating one while keeping the crossing whose exchange yields the trivial knot. Since a reduced alternating diagram must have the minimum number of crossings, by Kauffman, Murasugi $[\mathrm{M}]$ and Thistlethwaite $[\mathrm{Th}]$, we have the following.

Theorem A. For a certain minimum crossing diagram of an unknottingnumber-one two-bridge knot, there exist crossing whose exchange yields the trivial knot.

By the third Tait conjecture, all knot diagrams with the minimum crossings are transformed by a finite sequence of flypes. Here, a croissing whose exchange yields the trivial knot survives after flyping. Hence, we have the required result:

Theorem B. For every minimum crossing diagram of any given unknotting-number-one two-bridge knot, there exist crossings whose exchange yields the trivial knot, up to the third Tait conjecture (i.e. if his conjecture turns to be true).

\section{Deformation and Proof}

Proposition 1([KM]). The unknotting-number-one two-bridge knots can be represented in the Conway notation by $C\left(a, a_{1}, a_{2}, \cdots, a_{n}, \pm 2\right.$, $\left.-a_{n} \cdots,-a_{2},-a_{1}\right)$.

By the proposition above, an unknotting-number-one two-bridge knot can be illustrated as in Fig. 2. Here, $a, a_{2}, a_{4}, \cdots$ mean numbers of right-half-twist and $a_{1}, a_{3}, \cdots$ mean numbers of left-half-twist. The Fig. 2 shows the case $a=3, a_{1}=2, a_{2}=3, \therefore$. And a single exchange at each crossing marked by an asterisk yields the trivial knot.

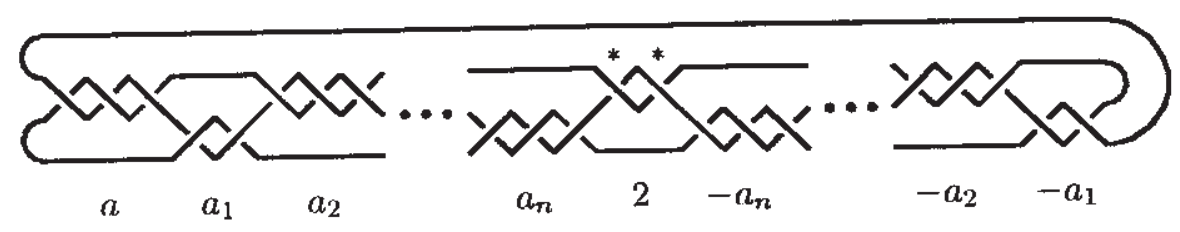

Fig. 2

In this section, we deform the diagram above into an alternating diagram. 
In the first step, we deform a diagram so that every pair of integers $a_{i}, a_{i+1}(1 \leq i \leq n-1)$ has the same sign as in Fig. 3. We rotate by $\pi$ the central part of three strings from the second crossing corresponding to $a_{i+1}$ through the second last crossing corresponding to $-a_{i+1}$ and the number of crossings is decreased by two with preserving a two-bridge form and the crossings marked by the asterisks.

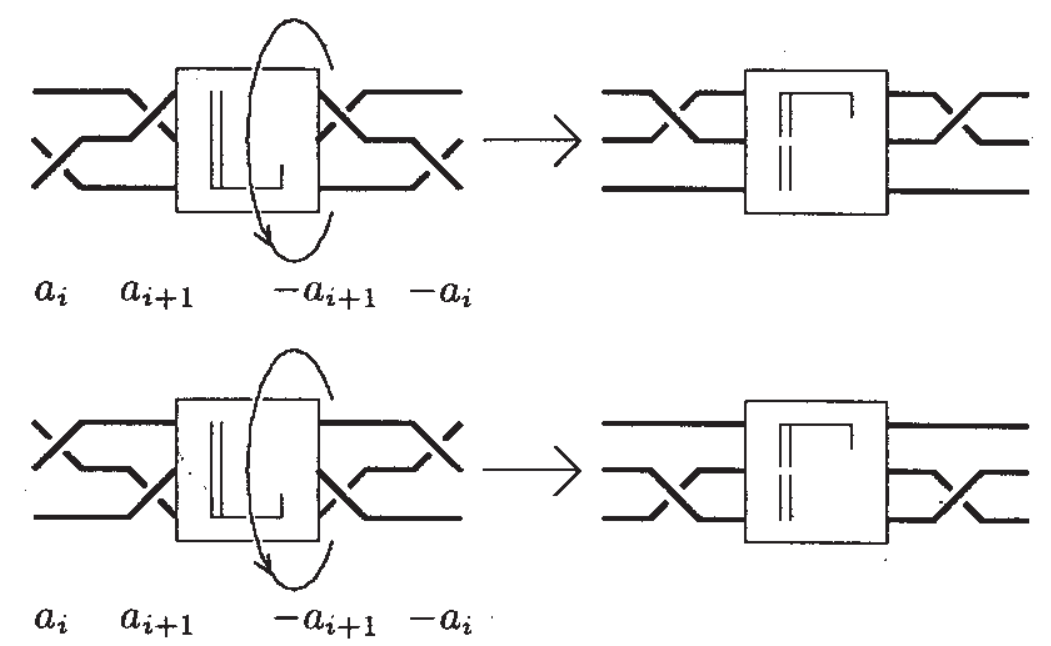

Fig. 3

Since the number of crossings is finite, a diagram can be deformed so that every pair of integers $a_{i}, a_{i+1}(1 \leq i \leq n-1)$ has the same sign by a finite sequence of operations in the first step.

In the second step, we deform a diagram into an alternating diagram with preserving the crossings marked by the asterisks. There are at most two possibilities of non-alternating after the first step; the pair of $a$ and 
$a_{1}$, and either one of the pair of $a_{n}$ and \pm 2 or the pair of \pm 2 and $-a_{n}$.

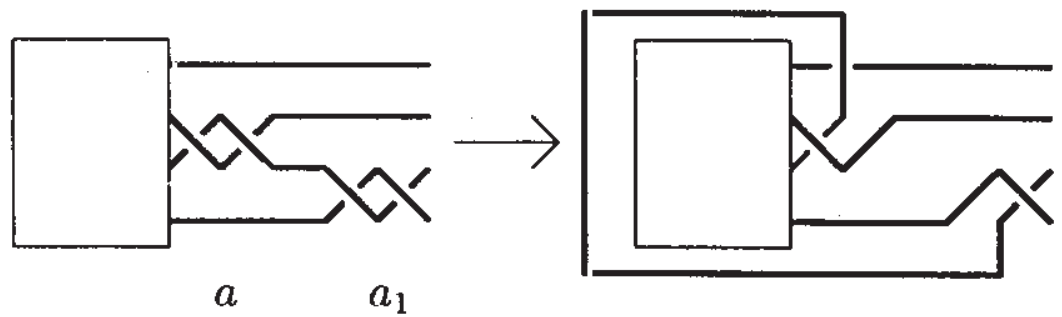

Fig. 4

For the former case, we push out the subarc from the last crossing corresponding to $a$ through the first crossing corresponding to $a_{1}$ as in Fig. 4.

For the latter case, we push out the subarc from the last crossing corresponding to $a_{n}$ through the first crossing corresponding to \pm 2 (or the subarc from the last crossing corresponding to \pm 2 through the first 
crossing corresponding to $-a_{n}$ ) as in Fig. 5 .

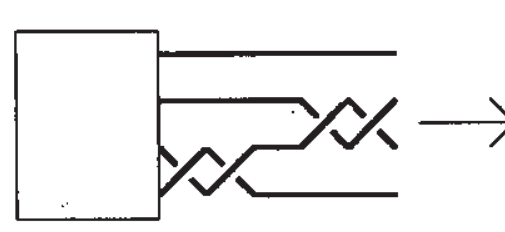

$a_{n} \quad-2$

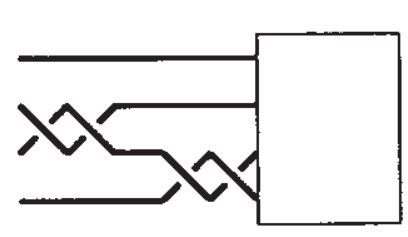

$2-a_{n}$
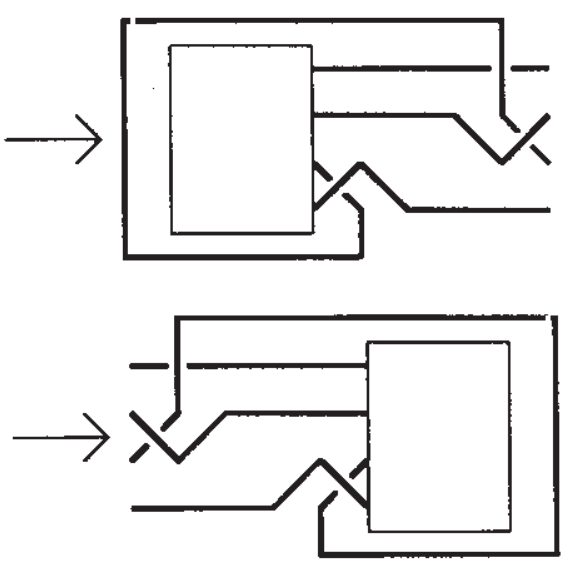

Fig. 5

The diagram after the second step is an alternating diagram and it is already reduced. By the following result by Kauffman, Murasugi [M] and Thistlethwaite [Th], it is a minimum crossing diagram and it has at least one crossing whose exchange yield the trivial knot.

Proposition 2 (The second Tait conjecture [M], [Th]). Two (connected and proper) alternating diagrams of an alternating knot have the same number of crossings. 
A flype is defined to be an operation on knot diagram as in Fig. 6. Here we remarked that the results of exchanges of the crossings marked by the asterisks have the same knot type. So we can say a crossing whose exchange yields the trivial knot survives after flyping.

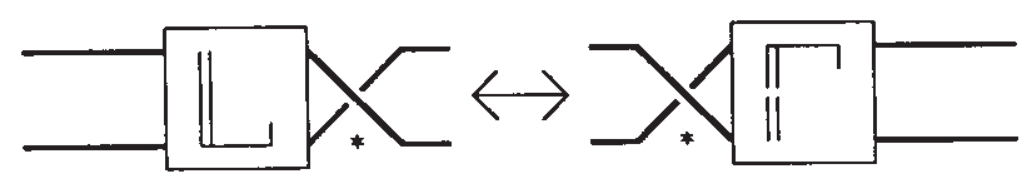

Fig. 6

Following the third Tait conjecture $[T]$, all reduced alternating diagrams of the same knot type can be deformed into each other by a finite sequence of flypes. Hence, there exist crossings whose exchange yields the trivial knot on every minimum crossing diagram of an unknottingnumber-one two-bridge knot.

Acknowledgement. The author would like to express his gratitude to Professor J. M. Montesinos for his suggestions and showing English correction.

\section{References}

[B] S. A. Bleiler, A note on unknotting number, Math. Proc. Cambridge Phils. Soc. 96 (1984), 469-471.

[C] J. H. Conway, An enumeration of krot and links, and their algebraic properties, Computational problem in Abstract Algebra (Oxford 1967), Pergamon Press, 1970, pp. 329-358.

[KM] T. Kanenobu and H. Murakami, Two-bridge knots with unknotting number one, Proc. Amer. Math. Soc 98 (1986), 499-502. 
[M] K. Murasugi, Jones polynomials and classical conjectures in knot theory, Topology 26 (1987), 187-194.

[N1] Y. Nakanishi, Unknotting numbers and knot diagrams with the minimum crossings, Math. Seminar Notes Kobe Univ. 11 (1983), 257258.

[N2] Y. Nakanishi, Union and tangle, to appear in Proc. Amer. Math. Soc.

[T] P. G. Tait, On knots, Scientific paper I, Cambridge Univ. Press, London, 1989, pp. 273-347.

[Th] M. B. Thistlethwaite, A spanning tree expansion of the Jones polynomial, Topology 26 (1987), 297-309.

Department of Mathematics

Recibido: 16 de Marzo de 1995

Kobe University

Nada-ku, Kobe, 657 Japan

E-mail addres: nakanisi@math.s.kobe-u.ac.jp 